\title{
The Measurement of the Refractive Index $n$ and $k$ Value of the EUV Resist by EUV Reflectivity Measurement Method
}

\author{
Yosuke Ohta ${ }^{*}$, Atsushi Sekiguchi ${ }^{1}$, Tetsuo Harada ${ }^{2}$, and Takeo Watanabe ${ }^{2}$ \\ ${ }^{1}$ Litho Tech Japan Co., Ltd., 2-6-6 Namiki, Kawaguchi, Saitama 332-0034, Japan \\ ${ }^{2}$ Center for EUV Lithography, Laboratory of Advanced Science and Technology for Industry, \\ University of Hyogo, 1-1-2 NewSUBARU, Kouto Kamigori, Ako-gun, Hyogo 678-1205, Japan \\ *ohta.yosuke@ltj.co.jp
}

\begin{abstract}
Up to now, we have been researching methods for measuring the simulation parameters of EUV resist. These parameters include the development parameter, the Dill C parameter, the diffusion length of acid generated from PAG, and the deprotection reaction parameter. By using these parameters, we have attempted to simulate EUV resist. As a result, we could investigate the conditions for reducing LER and for enhancing resolution. We hereby report on the methods of calculating the refractive index $n$ and $k$ values of photoresist with EUV light $(13.5 \mathrm{~nm})$, which has been difficult to measure until now, and the Dill B parameter, which is an absorption parameter. The three types of photoresists we investigated are the main chain scission type resist, chemically amplified resist, and metal resist.
\end{abstract}

Keywords: EUV reflectance measurement method, Refractive index, Dill B parameter, Main chain scission type resist, EUV chemically amplified resist, Metal resist

\section{Introduction}

As candidates for next-generation lithography with a half pitch (hp) of $15 \mathrm{~nm}$ or below, the ArF immersion multi-patterning technique and EUV lithography are the strongest. As it uses multiple masks and an etching technique, the multi-patterning technique is capable of obtaining high resolution, but its cost is high. On the other hand, the EUV lithography technique (hereinafter referred to as "EUVL") is advantageous in terms of cost because a single exposure is possible. Research on the photoresist suitable for EUV lithography requires cost and time. As a method for solving this issue, application of such photoresists to resist research using lithography simulation is useful. Up to now, we have studied the methods for measuring the simulation parameters of EUV resist.

These parameters include the development parameter [1], the Dill C parameter [2, 3], the diffusion length of acid generated from PAG [4], and the deprotection reaction parameter $[5,6]$. By using these parameters, we have attempted to simulate EUV resist. As a result, we could investigate the conditions for reducing LER and for enhancing resolution $[7,8]$. We hereby report on the methods of calculating the refractive index $\mathrm{n}$ and $\mathrm{k}$ values of photoresist with EUV light $(13.5 \mathrm{~nm})$, which has been difficult to measure until now, and the Dill B parameter, which is an absorption parameter, by focusing on the reflectance measurement method. The three types of

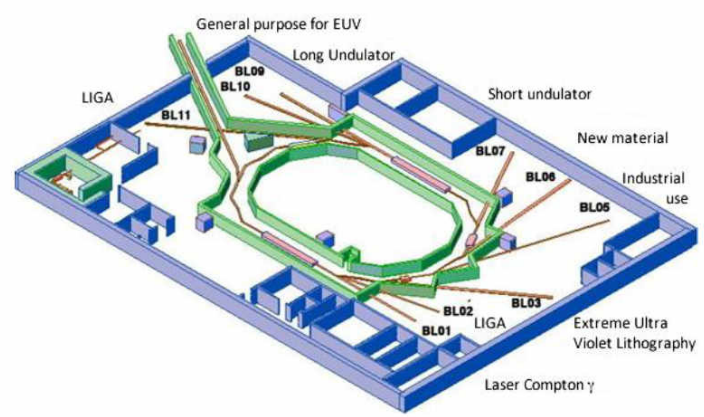

Fig. 1. NewSUBARU synchrotron radiation facility. 


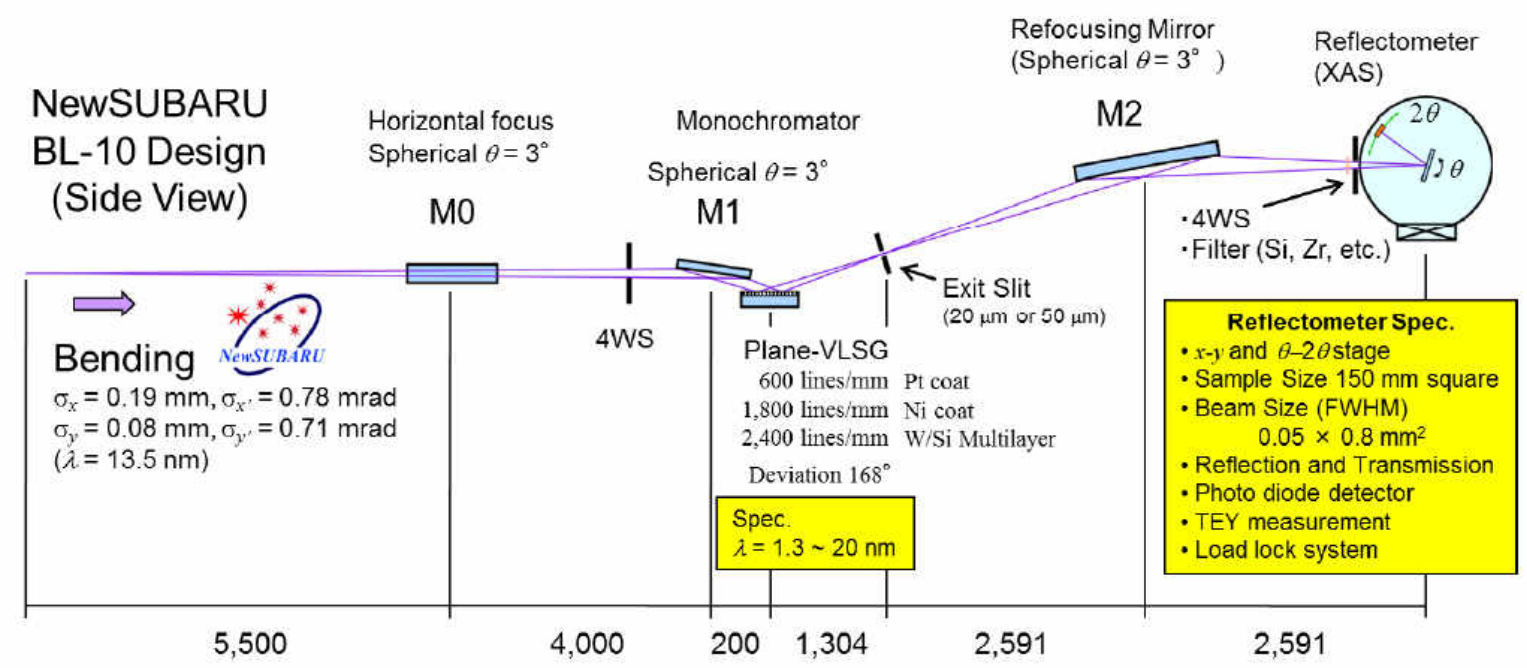

Fig. 2. Concept of reflectivity measurement system in BL-10.

photoresists for EUV we investigated are the main chain scission type resist, chemically amplified resist, and metal resist.

\section{Experiment equipment}

An overview of the reflection measurement system [9] in BL10 of the NewSUBARU Synchrotron Radiation Facility is provided in Figures 1 and 2. SR light emitted from a deflection magnet is monochromatized by a grating spectroscope to obtain EUV light with a wavelength of 13 to $14 \mathrm{~nm}$. By arranging a $250 \mathrm{~nm}$-thick silicone filter downstream of the grating, it is possible to irradiate the sample with EUV light.

Specifically, irradiated light is focused on the slit through the plane-VLSG by means of the M0 mirror horizontally and the M1 mirror vertically, and then focused horizontally and vertically on the measurement point in the chamber by means of the

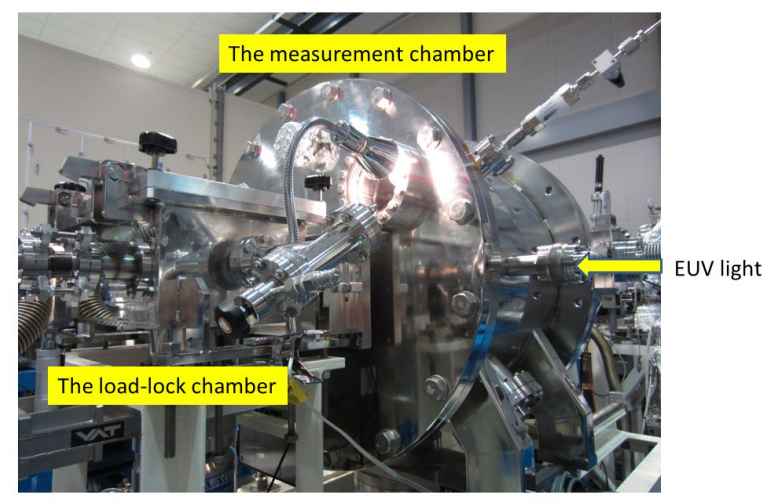

Fig. 3. Appearance of transmittance measurement system.
M2 mirror. The radiated light is then monochromatized into EUV light by this system and made usable for the experiment. The size of the focused beam is $0.8 \mathrm{~mm}(\mathrm{H}) \times 0.1 \mathrm{~mm}(\mathrm{~V})$.

Figure 3 shows an appearance of EUV reflectance measurement equipment. The intensity of the EUV light irradiated on the resist was measured by a photodiode.

\section{Measurement principle}

Figure 4 shows the measurement principle of the reflection angle and reflection intensity. A sample is placed on the rotary stage and irradiated with a $13.5 \mathrm{~nm}$-wavelength beam, then the reflection intensity is measured while the reflection angle is changed. An SXUV 100 is used as the detector photodiode, and the measurement condition is an oblique incidence angle in the range of 0 to $90^{\circ}$. By this method, it is possible to measure the reflection spectrum at a wavelength of $13.5 \mathrm{~nm}$. The

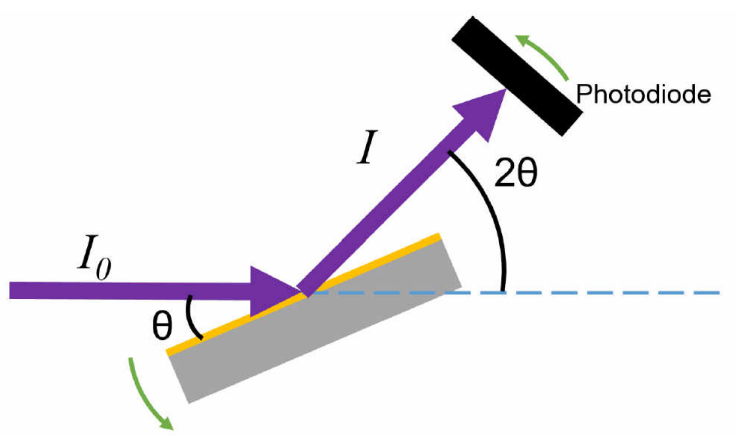

Fig. 4. Principle of reflection intensity measurement. 
reflectance $R$ can be obtained by Formula (1).

$$
R=\frac{I}{I_{0}}
$$

Where, $I$ represents reflection light intensity and $I_{0}$ represents incident light intensity.

\section{Experiment}

We measured the refractive indexes $n$ and $k$ of the main chain scission type electron beam resist ZEP520A (manufactured by Zeon Corporation), the acryl resin-based EUV resist SEVR-140 (manufactured by Shin-Etsu Chemical), and the $\mathrm{ZrO}_{2}$ metal resist (manufactured by Litho Tech Japan) with a wavelength of $13.5 \mathrm{~nm}$.

Resists were applied to Si substrates with film thickness of 25, 50, 75, and $100 \mathrm{~nm}$.

The process conditions are shown in Table 1.

Figure 5 and Figure 6 show the reflection
Table 1. Film information conditions.

\begin{tabular}{l|c|c|}
\hline \multirow{2}{*}{} & \multicolumn{2}{|c|}{ Pre-bake } \\
\cline { 2 - 3 } & Temperature $\left({ }^{\circ} \mathrm{C}\right)$ & Time $(\mathrm{s})$ \\
\hline ZEP-520A & 180 & 90 \\
\hline SEVR-140 & 130 & 90 \\
\hline ZrO2-MMA & 110 & 90 \\
\hline
\end{tabular}

spectrums of SEVR-140 and $\mathrm{ZrO}_{2}-\mathrm{MMA}$ resists by the reflection angles respectively.

In both measurements, we could confirm a fringe structure and obtain reflection angle spectrums. We calculated the $n$ and $k$ values by means of fitting. The fitting calculation to the measured spectrum was performed by IMD software program [10]. At that point, we conducted an analysis of the resist as a single-layer film, but this was not successful. Then, we employed a two-layer model, and the analysis was successful. With a two-layer model, we could observe the existence of a thin film layer with a different refractive index of 2 to $4 \mathrm{~nm}$ near the

\section{SEVR-140}
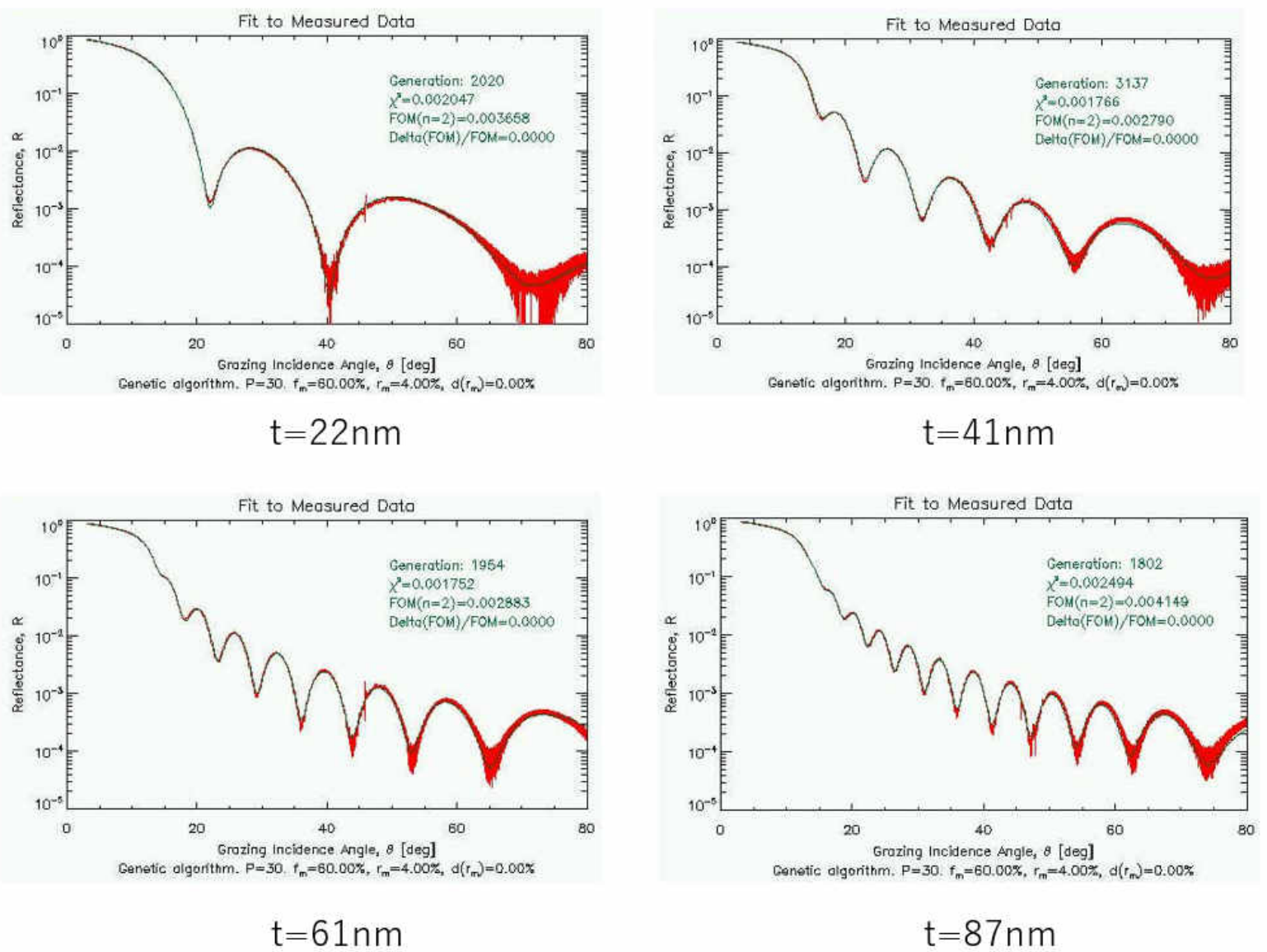

Fig. 5. Angle reflection spectrums of SEVR-140. 


\section{ZrO2-MMA}
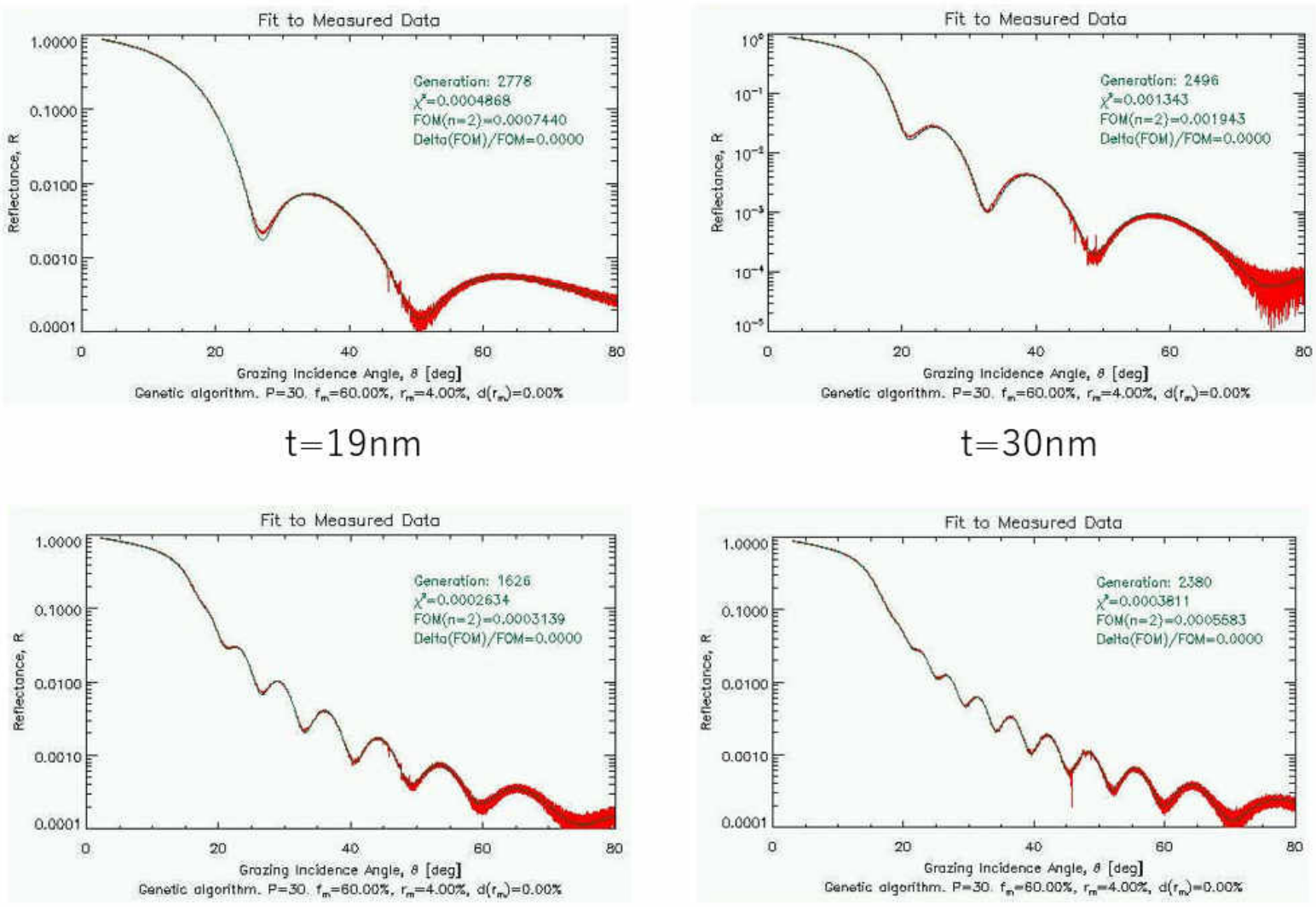

$$
\mathrm{t}=62 \mathrm{~nm}
$$

$$
\mathrm{t}=94 \mathrm{~nm}
$$

Fig. 6. Angle reflection spectrums of $\mathrm{ZrO}_{2}$-MMA.

substrate. Figure 7 shows the relationship between the refractive index $n$ and the film thickness in each resist.

The refractive index values $n$ of SEVR-140 and

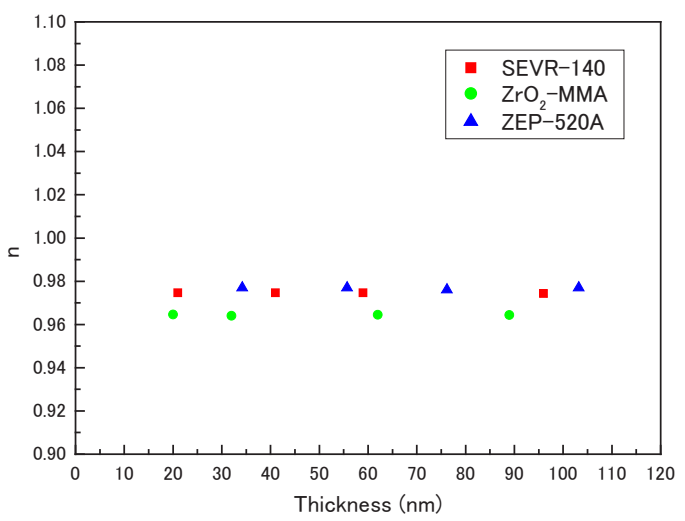

Fig. 7. Relationship between film thickness and refractive index.
ZEP-520A were similar, and the value of $\mathrm{ZrO}_{2-}$ MMA was slightly lower.

Figure 8 shows the relationship between the extinction coefficient $k$ and the film thickness in each resist.

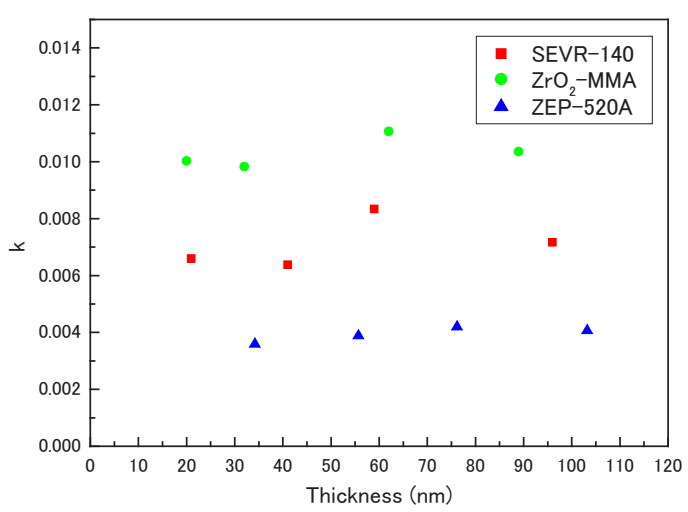

Fig. 8. Relationship between film thickness and extinction coefficient. 
The extinction coefficient $k$ was highest in $\mathrm{ZrO}_{2}-$ MMA, followed by SEVR-140 and ZEP-520A in that order. Concerning $\mathrm{ZrO}_{2}-\mathrm{MMA}$, it was confirmed that the refractive index $n$ is lower compared to polymer-based resist. As metal resists generally absorb EUV light, the extinction coefficient $\mathrm{k}$ in $\mathrm{ZrO}_{2}-\mathrm{MMA}$ is considered to be high [11].

\section{Calculation of absorption coefficient}

As the intensity of light is proportional to the square of the absolute value of the electric field amplitude, it is expressed by Formula (2).

$$
I \propto|E|^{2}=E_{O} 2 e^{\frac{-2 \omega \kappa x}{C}}
$$

Where, $\omega$ represents angular velocity, $k$ represents extinction coefficient, and $c$ represents light velocity. This formula indicates that when light travels through objects, the light attenuates as it is absorbed by the objects.

The absorption coefficient is a scale which is representing the strength of absorption by an object, and the change in this strength can be expressed by the Formula (3) in accordance with Lambert-Beer's law.

$$
I=I_{0} e^{-\alpha x}
$$

Where, $\alpha$ represents the absorption coefficient. The relationship between the absorption coefficient $\alpha$ and the extinction coefficient $k$ is expressed as Formula (4) in comparison with Formulas (2) and (3).

$$
\alpha=\frac{2 \omega k}{C}=\frac{4 \pi k}{\lambda}
$$

Where, $\lambda$ represents wavelength. The wavelength at this point is $13.5 \mathrm{~nm}$. Therefore, based on the extinction coefficient $k$, it is possible to calculate the absorption coefficient $\alpha$. At this point, when Formula (3) is deformed, it is possible to obtain Formula (5) which expresses the transmittance $T$.

$$
T=\frac{I}{I_{0}}=e^{-\alpha x}
$$

When the path length $x$ is equal to the film thickness $d$, the absorption coefficient $\alpha$ serves as the Dill B parameter and the parameter can be generally
Table 2. Measurement results of Dill B parameter.

\begin{tabular}{|c|c|}
\hline & $\mathrm{B}\left(\mu \mathrm{m}^{-1}\right)$ \\
\hline SEVR-140 & 6.14 \\
\hline ZrO2-MMA & 9.34 \\
\hline ZEP-520A & 3.75 \\
\hline
\end{tabular}

expressed by Formula (6) [12-15].

$$
B=-\frac{1}{d} \log T
$$

Table 2 shows the obtained Dill B parameters of each resists. When the Dill B parameters of respective resists were compared, the parameter of $\mathrm{ZrO}_{2}$-MMA is the highest, followed by those of SEVR-140 and ZEP-520A. This experiment also confirmed that metal resists have a higher absorption of EUV compared to polymer resists.

\section{Summary}

By using EUV light (13.5 nm), we measured the refractive indexes $n$ and extinction coefficients $k$ of resists by the reflectance measurement method. We also calculated the Dill B parameters based on the extinction coefficients.

As a result, we confirmed that the refractive index of metal resist $\mathrm{ZrO}_{2}-\mathrm{MMA}$ is lower than that of polymer resists and also confirmed that EUV light absorption by $\mathrm{ZrO}_{2}-\mathrm{MMA}$ is higher than that of polymer resists. This result is considered to be derived from the metal. In the future, we plan to measure $n$ and $k$ of metal resists containing $\mathrm{HfO}_{2}$ and $\mathrm{TeO}_{2}$ when EUV light is irradiated.

\section{Acknowledgements}

We gratefully acknowledge Mr. Motofumi Kashiwagi at Zeon Corporation and Mr. Jun Hatakeyama at Shin-Etsu Chemical for providing polymer materials.

\section{References}

1. A. Sekiguchi and Y. Sensu, Proc. SPIE, 8682 (2013) 55 .

2. A. Sekiguchi, Y. Matsumoto, H. Konishi, K. Moriyasu, and Y. Morimoto, Proc. SPIE, 7972 (2011).

3. A. Sekiguchi, Y. Matsumoto, and J. J. Biafor, Proc. SPIE, 8682 (2013).

4. A. Sekiguchi and Y. Matsumoto, Proc. SPIE, 9051 (2014). 
5. A. Sekiguchi, T. Watanabe, and H. Kinoshita, $J$. Photopolym. Sci. Technol., 27 (2014) 623.

6. A. Sekiguchi, Y. Kono, and Y. Sensu, $J$. Photopolym. Sci. Technol., 16 (2003) 209.

7. M. Yanagihara, J. Cao, M. Yamamoto, A. Arai, S. Nakayama, T. Mizuide, and T. Namioka, Applied Optics, 30 (1991) 2807.

8. M. Yoshifuji, S. Niihara, T. Harada, and T. Watanabe. Jpn. J. Appl. Phys., 58 (2019).

9. Y. Fukushima, T. Watanabe, T. Harada, and H. Kinoshita. J. Photopolym. Sci. Technol., 22 (2009) 85.

10. T. Ishiguro, J. Tanaka, T. Harada, and T. Watanabe. J. Photopolym. Sci. Technol., 32 (2019) 333.
11. B. Cardineau, R. D. Re, H. Al-Mashat, M. Marnell, M. Vockenhuber, Y. Ekinci, C. Sarma, M. Neisser, D. A. Freedman, and R. L. Brainard, Proc. SPIE, 9051 (2014).

12. A. Sekiguchi, T. Harada, and T. Watanabe, Proc. SPIE, 10143 (2017).

13. A. Sekiguchi, Y. Matsumoto, M. Isono, M. Naito, Y. Utsumi, T. Harada, and T. Watanabe, Proc. SPIE, 10583 (2018).

14. F. H. Dill, W. P. Hornberger, P. S. Hauge, and J. M. Shaw, IEEE Trans. Electron Devices, 22 (1975) 445.

15. F. H. Dill, IEEE Trans. Electron Devices, 22 (1975) 440. 